\title{
A Novel Algorithm for Fast BCG Cycle Extraction in Ambulatory Scenarios
}

\author{
Joan Gomez-Clapers, Ramon Casanella, Ramon Pallas-Areny \\ Universitat Politècnica de Catalunya - BarcelonaTech, Castelldefels, Spain
}

\begin{abstract}
A novel algorithm JDet for ballistocardiogram (BCG) cycle extraction is presented that is intended for the fast generation of ensemble averages in ambulatory scenarios where the ECG signal is not available. First, the sensitivity (Se) and positive predictivity $(+P)$ of JDet are evaluated on recordings obtained from 14 healthy subjects that stood on a modified weighing scale, and the results are compared with those from BSeg++, a popular BCG cycle-extraction algorithm. Second, the signal-tonoise ratio (SNR) is calculated for ensemble averages generated by using JDet, BSeg++, and the ECG R-waves on recordings of different duration. The results show that JDet has higher Se and similar $+P$ than BSeg++. Therefore, the quality of the ensemble averages generated from JDet can be comparable to those generated from the ECG, the only cost being a $25 \%$ longer recording time.
\end{abstract}

\section{Introduction}

The ballistocardiogram (BCG) is a recording of forces in the body that result from cardiac ejection and has lately attracted attention because it can deliver cardiovascular information in ambulatory scenarios such as homes or secondary medical facilities using simple and costeffective setups [1]. The BCG contains information of medical interest on its rate [2], power [3], amplitude [4], wave timings [5], and waveform shape [6].

Some BCG systems for continuous monitoring are embedded in furniture such as beds, chairs, or wheelchairs, but most efforts target the use of weighing scales or similar devices, which enable fast and periodic health status checks in ambulatory scenarios. However, BCG recordings from standing subjects often suffer from a high degree of motion artifacts caused by postural sway, muscular tremble, or respiration. Since these motion artifacts are usually in the same frequency band than the BCG, they cannot be removed by linear filters and need more advanced techniques.

A simple and common approach is to build ensemble averages from several heartbeats, generally segmented by using the $\mathrm{R}$ waves of a simultaneous auxiliary ECG for timing reference, which reduces noise by $1 / N, N$ being the number of heartbeats in the ensemble average. Several ensemble average sizes $(N)$ used to fit different requirements are 5 heartbeats [7][4], 9 heartbeats [8], or 15 seconds [9]. However, while the acquisition of the ECG in a laboratory under supervised conditions is simple and straightforward, its acquisition in ambulatory scenarios is often undesirable due to several drawbacks such as the increase in cost of the device, its worse integration in weighing scales, or the longer preparation time required that can hinder its incorporation to the subject's daily routine.

A plausible alternative is to obtain the segmentation points to build the ensemble average from the BCG signal itself, which can nowadays be performed with a wide variety of algorithms. However, algorithms that require a large number of heartbeats either for a training stage [10] or other purposes [11] hinder fast measurements. Among the algorithms that can operate with short recordings, wavelet-based algorithms [12] perform poorly when the heart rate has a wide range due to their narrow bandwidth response hence often need supervision or training to adjust the wavelet scales. Further, algorithms based on detecting the $\mathrm{J}$ wave, the most prominent BCG feature [13], are heavily influenced by motion artifacts and therefore do not suit ambulatory measurements. The remaining algorithms are mostly based on detecting the BCG envelope and, for the moment being, show the best performance on short recordings obtained in ambulatory scenarios.

BSeg++ [14] is a typical algorithm based on envelope detection that has evolved from two previous versions. However, our early trials revealed that its sensitivity (Se) decreases when it is applied to unsupervised BCG measurements from standing subjects, which requires longer recording times in order to generate ensemble averages of quality similar to that of those obtained by using the ECG R-wave for segmentation. Further, the possible effect of the positive predictivity $(+P)$ on the quality of the ensemble averages must be considered.

In this study, we propose a novel algorithm for BCG cycle extraction, JDet, which is based on a modified version of BSeg++ and is intended to particularly suit fast measurements in ambulatory scenarios where the ECG signal is not available. To assess its performance, both Se and $+P$ of JDet and BSeg ++ are evaluated for 14 subjects 
that stood unsupervised on a modified weighing scale. Additionally, the signal-to-noise ratio (SNR) of the ensemble averages generated with JDet, the ECG R-wave and BSeg++ for different recording durations are also compared in order to assess the suitability of JDet for fast measurements.

\section{Materials and methods}

\subsection{Signal acquisition system}

The four strain gages in an electronic weighing scale were connected into a Wheatstone bridge and interfaced to a differential amplifier by using a fully-differential first-order high-pass passive filter, with corner frequency set to $0.5 \mathrm{~Hz}$ [15] to reject the dc signal from body weight and low-frequency motion artifacts. Input filter resistors were selected large enough as compared to the strain gages to limit the voltage loading effect to less than $0.2 \%$.

The gain of the instrumentation amplifier was set to 5,000 , which yields an output offset of a few hundred millivolts removed by an ac-coupled amplifier with gain 5 and corner frequency set to $0.5 \mathrm{~Hz}$. The output is connected to a first-order low-pass filter with corner frequency set to $25 \mathrm{~Hz}$ [15] to reduce high-frequency noise and power line interference. The equivalent output impedance of this filter is small enough as compared to the input impedance of the ensuing data acquisition system.

The ECG was simultaneously recorded from a custom ambulatory ECG system [16] connected to the hands with adhesive wet-gel electrodes.

The sensors were interfaced to a computer via a data acquisition system (MicroDAQ-Lite, Eagle Technology, Cape Town, South Africa) configured to collect data from each channel at $1 \mathrm{kHz}$ sampling frequency.

\subsection{Experimental setup}

The signals were recorded from fourteen healthy volunteers without any history of cardiovascular diseases (age: 34 \pm 11 years, range: 22 years to 60 years; gender: 11 male, 3 female; weight: $65 \pm 16 \mathrm{~kg}$, range: $53 \mathrm{~kg}$ to $81 \mathrm{~kg}$; height: $174 \pm 8 \mathrm{~cm}$, range: $160 \mathrm{~cm}$ to $189 \mathrm{~cm}$ ) that gave their informed consent.

Two ECG electrodes were fixed each on the back of one hand and the subjects were asked to stand still in the modified weighing scale for $50 \mathrm{~s}$ while the signals were being recorded.

\subsection{Proposed algorithm}

The JDet approach is similar to that of BSeg++ but some steps have been tuned to maximize motion-artifact rejection yet preserving most of the BCG signal power. The main changes are in the frequency and order of the filters, which are higher and narrower to reject frequency bands where motion artifacts predominate over the BCG, and in the use of the BCG power to detect the envelope instead of detecting the BCG signal itself. Further, filters are applied forward and reverse to avoid time shifts of BCG features due to phase distortion.

The algorithm has the following steps:

1) Motion artifacts are rejected while most of the BCG power is preserved by filtering the signal with a $1^{\text {st }}$ order band-pass filter with cutoff frequencies $2 \mathrm{~Hz}$ and $7 \mathrm{~Hz}$, applied forward and reverse.

2) The signal envelope is extracted by filtering the power of the BCG with a $2^{\text {nd }}$ order low-pass Butterworth filter with cutoff frequency $2 \mathrm{~Hz}$, applied forward and reverse.

3) Individual heartbeats are detected from the peaks in the envelope signal.

4) When two detected peaks are closer than $400 \mathrm{~ms}$, the peak with lower amplitude is removed. This limits the maximum heart rate up to 150 beats per minute, which is reasonably high for standing healthy subjects.

5) For each heartbeat, the maximum peak of the original BCG signal in a $100 \mathrm{~ms}$ interval centered in the maximum of the envelope is classified as a $\mathrm{J}$ wave, which is the most prominent positive peak of the signal.

\subsection{Signal processing and data analysis}

JDet was evaluated by comparing its timing references to those obtained from the ECG. $\mathrm{R}$ waves were first detected in ECG recordings by using the Pan-Tompkins algorithm [17] and the results were manually inspected to guarantee the absence of false positives (FP) and false negatives (FN). J waves in an interval of 120 ms centered at the time point $211 \mathrm{~ms}$ after the $\mathrm{R}$ wave, where a $99 \%$ $(3 \sigma)$ of $\mathrm{J}$ waves are expected to be found [4], were classified as true positives (TP). The remaining $\mathrm{J}$ waves were classified as FP whereas intervals after the $\mathrm{R}$ wave without a J wave were classified as FN. BSeg++ was also evaluated for reference using the same criteria.

The SNR of each recording, termed $S_{N R}$, was measured by dividing it in two subsets of equal duration (25s) to avoid the excessive filtering of high-frequency features due to small changes in the BCG waveform induced by hemodynamic regulation during the experiment. For each subset, the individual heartbeats $x_{\mathrm{i}}$ were identified and segmented in samples $500 \mathrm{~ms}$ long starting from the ECG R-wave and an ensemble average $\bar{x}_{\text {ECG }}$ was built from it. The SNR of each individual heartbeat $\mathrm{SNR}_{i}$ was calculated as 


$$
\operatorname{SNR}_{i}=\frac{\operatorname{var}\left(\bar{x}_{\mathrm{ECG}}\right)}{\operatorname{var}\left(x_{i}-\bar{x}_{\mathrm{ECG}}\right)},
$$

and $\mathrm{SNR}_{\mathrm{R}}$ was calculated as the average of the individual $\mathrm{SNR}_{i}$ in the two subsets.

The quality of the ensemble averages generated from JDet was evaluated from their SNR calculated with respect to ECG-generated ensemble averages to assess the effect of FP and FN for different recording durations. The same as in (1), the original recordings were split in two subsets to avoid the effect of minor changes in the BCG waveform. The effect of the recording duration was assessed by comparing the ensemble averages $\bar{x}_{\mathrm{ECG}}$ generated from the ECG R-waves found in the whole subset to the ensemble averages $\bar{x}_{t}$ generated only with heartbeats found in the subinterval $[0, t]$. The SNR of the ensemble average for a certain subinterval $\mathrm{SNR}_{t}$ was calculated as

$$
\mathrm{SNR}_{t}=\frac{\operatorname{var}\left(\bar{x}_{\mathrm{ECG}}\right)}{\operatorname{var}\left(\bar{x}_{t}-\bar{x}_{\mathrm{ECG}}\right)} .
$$

$\mathrm{SNR}_{t}$ was calculated for the ECG R-wave, JDet and BSeg++ using the same methodology to provide reference values for several interval durations from $2 \mathrm{~s}$ to $20 \mathrm{~s}$. For JDet an BSeg++, BCG samples were segmented starting from the $\mathrm{J}$ wave minus the $\mathrm{RJ}$ duration to assure a proper alignment with ECG-generated ensemble averages.

\section{Results}

The characterization of JDet and BSeg++ in our data set, summarized in Table 1, show that a poor SNR is directly related to a high number of FN and FP. Se and $+P$ values are respectively $0.87 \%$ and $0.92 \%$ for the proposed algorithm and $0.57 \%$ and $0.87 \%$ for BSeg++.

Table 1. Evaluation of JDet and BSeg++.

\begin{tabular}{lrrrrrr}
\hline \multirow{2}{*}{ Rec. } & \multirow{2}{*}{ \# } & \multicolumn{2}{c}{ JDet } & \multicolumn{2}{c}{ BSeg++ } & SNR $_{\text {R }}$ \\
\cline { 3 - 6 } & beats & FN & FP & FN & FP & $($ dB $)$ \\
\hline S1 & 60 & 0 & 0 & 17 & 0 & 13.8 \\
S2 & 49 & 3 & 3 & 5 & 2 & 12.8 \\
S3 & 55 & 18 & 12 & 38 & 8 & 2.7 \\
S4 & 62 & 5 & 4 & 26 & 7 & 7.1 \\
S5 & 49 & 0 & 0 & 5 & 1 & 17.8 \\
S6 & 59 & 6 & 8 & 27 & 5 & 3.8 \\
S7 & 51 & 0 & 0 & 7 & 0 & 13.2 \\
S8 & 54 & 12 & 9 & 39 & 11 & 2.6 \\
S9 & 82 & 19 & 7 & 52 & 4 & 7.4 \\
S10 & 48 & 0 & 0 & 16 & 2 & 11.2 \\
S11 & 47 & 4 & 4 & 19 & 9 & 12.1 \\
S12 & 82 & 30 & 13 & 57 & 9 & 3.5 \\
S13 & 50 & 10 & 7 & 16 & 8 & 7.7 \\
S14 & 59 & 0 & 0 & 19 & 2 & 9.7 \\
\hline Total & 807 & 107 & 67 & 343 & 68 & 10.1 \\
\hline
\end{tabular}

The main advantage of the proposed algorithm is a $70 \%$ reduction of FN while the number of FP is about the same. This can represent a significant reduction in the time required to generate an ensemble average of a certain number of heartbeats, especially for recordings that have a high number of FN (above a $50 \%$ of the total) such as S3, S8, S9, or S12.

Figure 1 shows the mean SNR achieved for all subjects by the three methods for different recording durations. It can be observed that ECG-generated ensemble averages show the fastest increase in SNR with the recording duration and achieve the maximal values due to the lack of FP and FN, as these are only influenced by motion artifacts in BCG recordings. BSeg++ has the worse Se and $+P$ and therefore its SNR is the worse of the three methods for all recording durations. JDet has higher SNR than BSeg++, especially for short recordings due to its superior $S e$, but its SNR for long durations is limited by FP and trends towards a value similar to that of BSeg++. For short recording durations, the recording length required to achieve a given SNR by JDet is about $25 \%$ longer than that when using the ECG, whereas BSeg++ needs recordings about $100 \%$ longer.

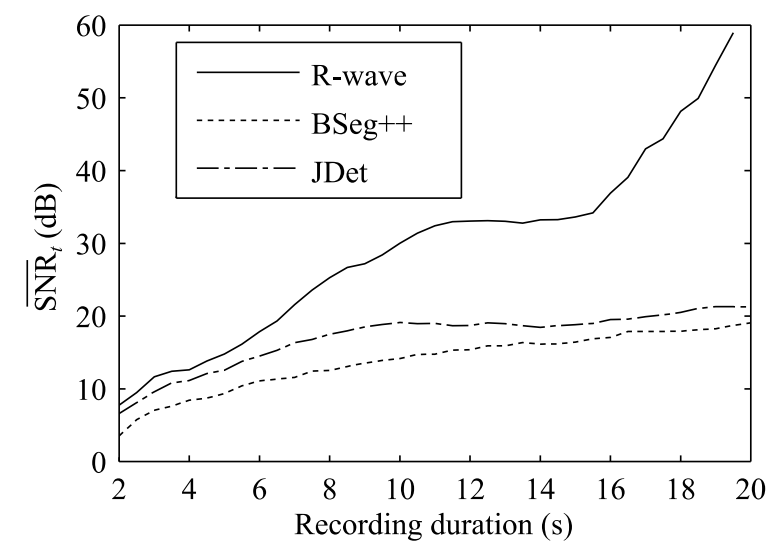

Figure 1. Mean SNR of BCG ensemble averages for all subjects with respect to the recording duration.

These results show that, in general, the minimum recording duration for a given SNR is mostly influenced by the $S e$ value of the algorithm whereas the $+P$ value limits the maximal SNR that can be achieved.

\section{Conclusions}

A novel algorithm JDet has been proposed that is able to quickly extract BCG cycles when no ECG reference is available, as it is often the case in ambulatory scenarios. In measurements obtained from 14 subjects that stood on a modified weighing scale, JDet has shown a $S e$ value of $0.87 \%$ and $+P$ value of $0.92 \%$, which outperforms BSeg++, a popular BCG cycle extraction algorithm that 
does not need a reference ECG either. As a result, in the range of recording times where the three algorithms can be compared, the recording duration required by JDet to achieve a given SNR value in an ensemble average is only about $25 \%$ longer than when cycle extraction is based on the ECG R-wave, whereas BSeg++ needs recordings about $100 \%$ longer.

Even though the comparison has been performed for a reduced and uniform cohort, the results provide guidelines for further algorithms intended for short recordings in larger, heterogeneous cohorts in ambulatory scenarios. Their application to widely used electronic weighing scales can enable alternative methods for quickly obtaining cardiovascular parameters at home or in primary care centers.

\section{Acknowledgements}

The authors want to thank the volunteers for their valuable cooperation and F. Lopez for his technical support.

\section{References}

[1] O. T. Inan, P. F. Migeotte, K.-S. Park, M. Etemadi, K. Tavakolian, et al. Ballistocardiography and Seismocardiography: A Review of Recent Advances. Biomed. Heal. Informatics, IEEE J. 2015; 19(4): 1414-27.

[2] R. Pallas-Areny, R. Gonzalez-Landaeta, O. Casas. Heart Rate Detection from an Electronic Weighing Scale. Physiol. Meas. 2008; 29(8): 979-88.

[3] O. T. Inan, M. Etemadi, A. Paloma, L. Giovangrandi, G. T. A. Kovacs. Non-invasive Cardiac Output Trending During Exercise Recovery on a Bathroom-scale-based Ballistocardiograph. Physiol. Meas. 2009; 30(3): 261-74.

[4] O. T. Inan, M. Etemadi, R. M. Wiard, L. Giovangrandi, G. T. A. Kovacs. Robust Ballistocardiogram Acquisition for Home Monitoring. Physiol. Meas. 2009; 30(2): 169-85.

[5] M. Etemadi, O. T. Inan, L. Giovangrandi, G. T. A. Kovacs. Rapid Assessment of Cardiac Contractility on a Home Bathroom Scale. Inf. Technol. Biomed. IEEE Trans. 2011; 15(6): 864-69.

[6] H. Mandelbaum, R. A. Mandelbaum. Studies Utilizing the Portable Electromagnetic Ballistocardiograph. I. Abnormal HIJK Patterns in Hypertensive and Coronary Artery Heart Disease. Circulation. 1951; 3(5): 663-73.

[7] J. H. Shin, K. M. Lee, K. S. Park. Non-constrained Monitoring of Systolic Blood Pressure on a Weighing Scale. Physiol. Meas. 2009; 30(7): 679-93.

[8] A. D. Wiens, O. T. Inan. Accelerometer Body Sensor Network Improves Systolic Time Interval Assessment with Wearable Ballistocardiography. Eng. Med. Biol. Soc. (EMBS), 2015. 37th Annu. Int. Conf. 2015: 1833-36.

[9] O. T. Inan, M. Etemadi, R. M. Wiard, G. T. A. Kovacs, L. Giovangrandi. Non-invasive Measurement of Valsalvainduced Hemodynamic Changes on a Bathroom Scale Ballistocardiograph. Eng. Med. Biol. Soc. (EMBS), 2008. 30th Annu. Int. Conf. IEEE. 2008: 674-7.

[10] C. Bruser, K. Stadlthanner, S. de Waele, S. Leonhardt.
Adaptive Beat-to-Beat Heart Rate Estimation in Ballistocardiograms. Inf. Technol. Biomed. IEEE Trans. 2011; 15(5); 778-86.

[11] J. Paalasmaa, H. Toivonen, M. Partinen. Adaptive Heartbeat Modeling for Beat-to-Beat Heart Rate Measurement in Ballistocardiograms. Biomed. Heal. Informatics, IEEE J. 2015; 19(6): 1945-52.

[12] O. A. Postolache, P. M. B. S. Girao, J. Mendes, E. C. Pinheiro, G. Postolache. Physiological Parameters Measurement Based on Wheelchair Embedded Sensors and Advanced Signal Processing. Instrum. Meas. IEEE Trans. 2010; 59(10): 2564-74.

[13] B. H. Choi, G. S. Chung, J.-S. Lee, D.-U. Jeong, K. S. Park. Slow-wave Sleep Estimation on a Load-cell-installed Bed: A Non-constrained Method. Physiol. Meas. 2009; 30(11): 1163-70.

[14] A. Akhbardeh, B. Kaminska, K. Tavakolian. BSeg++: A modified Blind Segmentation Method for Ballistocardiogram Cycle Extraction. Eng. Med. Biol. Soc. (EMBS), 2007. 29th Annu. Int. Conf. IEEE. 2007: 189699.

[15] J. Gomez-Clapers, A. Serra-Rocamora, R. Casanella, R. Pallas-Areny. Towards the Standardization of Ballistocardiography Systems for J-peak Timing Measurement. Measurement. 2014; 58:310-16.

[16] J. Gomez-Clapers, R. Casanella. A Fast and Easy-to-use ECG Acquisition and Heart Rate Monitoring System Using a Wireless Steering Wheel. Sensors Journal, IEEE. 2012; 12(3): 610-16.

[17] J. Pan, W. J. Tompkins. A Real-time QRS Detection Algorithm. Biomed. Eng. IEEE Trans. 1985; 32(3): 23036.

Address for correspondence:

Joan Gómez Clapers

EETAC-UPC

c/Esteve Terradas 7, edif. C4, lab. 123.

08860 Castelldefels (Spain)

joan.gomez-clapers@upc.edu 